Homology, Homotopy and Applications, vol.8(1), 2006, pp.257-261

\title{
FIBRATIONS OVER ASPHERICAL MANIFOLDS
}

\section{DACIBERG GONÇALVES AND PETER WONG}

(communicated by James Stasheff)

Abstract

Let $f: E \rightarrow B$ be a map between closed connected orientable manifolds. In this note, we give a necessary condition for $f$ to be a manifold fibration. In particular, we show that if $F \hookrightarrow E \stackrel{f}{\rightarrow} B$ is a fibration where $F=f^{-1}(b), E$ and $B$ are closed connected triangulated orientable manifolds and $B$ is aspherical, then $\left.f\right|_{E^{(n)}}: E^{(n)} \rightarrow B$ is surjective, where $E^{(n)}$ denotes the $n$-th skeleton of $E$ and $n=\operatorname{dim} B$.

Dedicated to Professor Ed Fadell

Fibrations are fundamental objects of study in topology and in other areas of mathematics. Given a surjective map $f: E \rightarrow B$, it is natural to ask if the homotopy class $[f]$ contains a representative $f^{\prime}$ that is a fibration. Farrell in [4], upon improving his previous results, gave an obstruction for a smooth map $f: M \rightarrow S^{1}$ to be a fiber bundle over the circle $S^{1}$. Our objective here is to consider this problem from the point of view of coincidence theory involving aspherical manifolds as the target space.

Let $f, g: M \rightarrow N$ be maps between two closed connected manifolds. Coincidence theory is concerned with the study of the coincidence set $C(f, g)=\{x \in M \mid f(x)=$ $g(x)\}$. When $g=\bar{b}$ is the constant map at a point $b \in N$, the coincidence problem becomes the study of preimages of a point under $f$. In [5], the primary obstruction to deforming $f$ and $g$ to be coincidence free on the $n$-th skeleton of $M, n=\operatorname{dim} N$, was defined and studied. In this note, we study the preimage problem, i.e., when $g=\bar{b}$. In particular, we show that this primary obstruction is non-trivial when $f$ is a fibration and $B=N$ is aspherical. This implies that a typical fiber must intersect the $n$-th skeleton of the total space $E$, regardless of the cellular decomposition of $E$. This provides more information about (the fiber of) such fibrations.

\section{Primary obstruction to deformation}

Let $f, g: M \rightarrow N$ be maps between two closed connected orientable manifolds with $\operatorname{dim} M \geqslant \operatorname{dim} N=n$. We write $\pi_{M}=\pi_{1}(M), \pi_{N}=\pi_{1}(N)$. Then, $\left[\pi_{n}\left(N^{\times}\right)\right]_{a b}$ is a local system on $N \times N$, where $N^{\times}$denotes the pair $\left(N \times N, N \times N-\Delta_{N}\right)$ and

Received December 13, 2005, revised January 3, 2006; published on February 1, 2006.

2000 Mathematics Subject Classification: Primary: 55M20, 55R20, 55T10; Secondary: 55S35.

Key words and phrases: Obstruction theory, fibrations, local coefficients, Shapiro's Lemma.

Copyright (C) 2006, International Press. Permission to copy for private use granted. 
$[G]_{a b}$ denotes the abelianization of a group $G$. Similar to the definition in $[\mathbf{5}]$, we define the twisted Thom class $\tilde{\tau}_{N} \in H^{n}\left(N^{\times} ;\left[\pi_{n}\left(N^{\times}\right)\right]_{a b}\right)$ to be the cohomology class of the cocycle $u_{N} \in C^{n}\left(N^{\times} ;\left[\pi_{n}\left(N^{\times}\right)\right]_{a b}\right)$ given by $\left\langle u_{N}, \sigma\right\rangle=[\sigma] \in \pi_{n}\left(N^{\times} ; \sigma\left(v_{0}\right)\right)$ for $\sigma:\left(\Delta_{n}, \partial \Delta_{n}\right) \rightarrow N^{\times}$, where $v_{0}$ is the leading vertex of the standard $n$-simplex $\Delta_{n}$. Denote by $\tau_{N}$ the ordinary Thom class of the normal bundle of the diagonal $\Delta_{N}$ in $N \times N$ using $\mathbb{Z}$ coefficients. If $n \geqslant 3$, then $\left[\pi_{n}\left(N^{\times}\right)\right]_{a b} \cong \pi_{n}\left(N^{\times}\right) \cong \mathbb{Z} \pi_{N}$. When $n=2, N$ is an orientable surface of genus $g \geqslant 0$. Note that $\left[\pi_{n}\left(N^{\times}\right)\right]_{a b} \cong$ $\left[\pi_{n}(N, N-b)\right]_{a b}$, where $b \in N$. Using the universal covering space of $N$ and the same argument as in [2], one can show that $\left[\pi_{2}(N, N-b)\right]_{a b} \cong \mathbb{Z} \pi_{N}$. Then the augmentation map $\mathbb{Z} \pi_{N} \rightarrow \mathbb{Z}$ induces $\epsilon^{*}: H^{n}\left(N^{\times} ; \mathbb{Z} \pi_{N}\right) \rightarrow H^{n}\left(N^{\times} ; \mathbb{Z}\right)$ such that $\epsilon^{*}\left(\tilde{\tau}_{N}\right)=\tau_{N}$.

The primary obstruction to deforming $f$ and $g$ to be coincidence free on the $n$-th skeleton is given by

$$
o_{n}(f, g):=[j(f \times g) d]^{*}\left(\tilde{\tau}_{N}\right) \in H^{n}\left(M ; \mathbb{Z} \pi_{N}^{*}\right),
$$

where $d$ is the diagonal map $M \rightarrow M \times M$ and $j$ is the inclusion of pairs $(N \times N, \emptyset) \rightarrow N^{\times}$. Here, $\mathbb{Z} \pi_{N}{ }^{*}$ denotes the local system over $M$ induced by pulling back the local system $\mathbb{Z} \pi_{N}$ along the map $j(f \times g) d$. Thus, for $n \geqslant 3, o_{n}(f, g)$ coincides with the primary obstruction defined in [5] whereas for $n=2, o_{2}(f, g)$ is the abelianized obstruction which coincides with that in $[\mathbf{3}]$ when $g$ is the identity and $M=N$. Note that if $M^{(n)}$ denotes the $n$-th skeleton of $M$ and $o_{n}(f, g) \neq 0$, then for any $f^{\prime} \sim f, g^{\prime} \sim g$ we have $M^{(n)} \cap C\left(f^{\prime}, g^{\prime}\right) \neq \emptyset$.

\section{Main results}

Let $p: E \rightarrow B$ be a fibration and let $F=p^{-1}(b), b \in B$ be a typical fiber. Suppose that $F, E$, and $B$ are 0 -connected closed triangulated orientable manifolds. We call such a fibration a manifold fibration.

Theorem 2.1. Let $p: E \rightarrow B$ be a manifold fibration with $\operatorname{dim} E=n+k$ and $\operatorname{dim} B=n$. Suppose that $\pi_{q}(B)=0$ for $2 \leqslant q \leqslant k+1$. Then for any $b \in B$, $p^{-1}(b) \cap E^{(n)} \neq \emptyset$. In other words, $\left.p\right|_{E^{(n)}}: E^{(n)} \rightarrow B$ is surjective. For $n \geqslant 2$, the primary obstruction $o_{n}(p, \bar{b})$ is a non-zero integer.

Proof. Case 1: $n=1$.

Here, $B=S^{1}$, the unit circle. If $p$ were deformable to a map $p^{\prime}$ such that $p^{\prime}$ does not have preimages in the 1-skeleton of $E$, then $p^{\prime}$, which we may assume is cellular, has the property that $p^{\prime} \mid E^{(1)}$ factors through $S^{1}-b$. Now, $\left(p^{\prime} \mid E^{(1)}\right)_{\#}: \pi_{1}\left(E^{(1)}\right) \rightarrow$ $\pi_{1}\left(S^{1}\right)$ is surjective. This is because the fiber is path connected so that $p_{\#}^{\prime}: \pi_{1}(E) \rightarrow$ $\pi_{1}\left(S^{1}\right)$ is surjective, and $\left(p^{\prime} \mid E^{(1)}\right)_{\#}: \pi_{1}\left(E^{(1)}\right) \rightarrow \pi_{1}(E)$ is also surjective. However, the factoring of $p^{\prime}$ through the contractible subspace $S^{1}-b$ yields a contradiction to the surjectivity of $\left(p^{\prime} \mid E^{(1)}\right)_{\#}$. Hence, the assertion follows.

Case 2: $n>1$.

Since $\pi_{2}(B)=0$, from the long exact sequence of homotopy groups of the 
fibration, we have a short exact sequence

$$
0 \rightarrow \pi_{1}(F) \stackrel{i_{\#}}{\rightarrow} \pi_{1}(E) \stackrel{p_{\#}}{\rightarrow} \pi_{1}(B) \rightarrow 1
$$

where $i: F \hookrightarrow E$ is the inclusion.

Let $\eta: E_{0} \rightarrow E$ be the covering space corresponding to the subgroup $i_{\#}\left(\pi_{1}(F)\right) \triangleleft$ $\pi_{1}(E)$. It follows from the topological analog for homology of Shapiro's Lemma [6] (see also the cohomology version in [1]) that

$$
H_{*}\left(E_{0} ; \mathbb{Z}\right) \cong H_{*}\left(E ; \mathbb{Z}\left[\pi_{B}^{*}\right]\right)=H_{*}\left(E ; \mathbb{Z}\left[p^{*} \pi_{B}\right]\right),
$$

where $\pi_{B} \cong \pi_{1}(E) / i_{\#}\left(\pi_{1}(F)\right)$ is the local coefficient system on $B$.

Now, the inclusion $i: F \hookrightarrow E$ lifts to $\tilde{i}: F \rightarrow E_{0}$ so that $i=\eta \circ \tilde{i}$. Since $\pi_{q}(B)=0$ for $2 \leqslant q \leqslant k+1$, it follows that $\tilde{i}: F \rightarrow E_{0}$ induces isomorphisms on $\pi_{q}$ for $1 \leqslant q \leqslant k$ and an epimorphism on $\pi_{k+1}$. Hence, $\tilde{i}_{*}: H_{k}(F ; \mathbb{Z}) \rightarrow H_{k}\left(E_{0} ; \mathbb{Z}\right)$ is an isomorphism. Now,

$$
H_{k}\left(E ; \mathbb{Z}\left[p^{*} \pi_{B}\right]\right) \cong H_{k}\left(E_{0} ; \mathbb{Z}\right) \stackrel{\tilde{i}_{*}^{-1}}{\rightarrow} H_{k}(F ; \mathbb{Z}) \cong \mathbb{Z}
$$

By Poincaré duality (see $[\mathbf{7}]$ ), we have

$$
H^{n}\left(E ; \mathbb{Z}\left[p^{*} \pi_{B}\right]\right) \cong H_{k}\left(E ; \mathbb{Z}\left[p^{*} \pi_{B}\right]\right) .
$$

It has been shown in [5] that the primary obstruction $o_{n}(f, g)$ is Poincaré dual to the twisted homology class corresponding to the coincidence submanifold of $f$ and $g$ (without loss of generality, we may assume that $f$ and $g$ are transverse). Although it was assumed in [5] that $n \geqslant 3$, the same argument there holds when $n=2$ provided the coefficients form a local system and thus we replace $\pi_{2}\left(N^{\times}\right)$ with its abelianization $\left[\pi_{2}\left(N^{\times}\right)\right]_{a b}$. With $f=p$ and $g=\bar{b}$, the primary obstruction $o_{n}(p, \bar{b}) \in H^{n}\left(E ; \mathbb{Z}\left[p^{*} \pi_{B}\right]\right)$ is Poincaré dual to the class $\left[z_{i(F)}^{p^{*} \pi_{B}}\right] \in H_{k}\left(E ; \mathbb{Z}\left[p^{*} \pi_{B}\right]\right)$, which, by $(1)$, corresponds to $\left[z_{F}^{i^{*} p^{*} \pi_{B}}\right]$, the fundamental class $\left[z_{F}\right]$ of $F$ in $H_{k}(F ; \mathbb{Z})$, since the coefficient system $i^{*} p^{*} \pi_{B}$ is trivial. Hence, $o_{n}(p, \bar{b})$ corresponds to $\left[z_{F}\right] \in \mathbb{Z}-\{0\}$.

Remark 2.2. Theorem 2.1 shows that $p^{-1}(b)$ must intersect the $n$-th skeleton $E^{(n)}$ of $E$, regardless of the cellular decomposition of $E$. In fact, any map homotopic to $p$ has the same property, that is, the set of preimages of $b$ must also intersect $E^{(n)}$. Furthermore, this result shows that the non-vanishing of the obstruction is a necessary condition for a map to be (homotopic to) a fibration.

Remark 2.3. In Theorem 2.1, the hypotheses on the higher homotopy groups of $B$ cannot be relaxed. The Hopf bundles $S^{2 n+1} \rightarrow \mathbb{C} P^{n}$ and $S^{4 n+3} \rightarrow \mathbb{H} P^{n}$ are easy counter-examples.

The following is an equivalent formulation of Theorem 2.1 when $B$ is aspherical.

Theorem 2.4. Given a map $f: E \rightarrow B$ from a closed connected triangulable oriented manifold to a closed connected triangulable oriented aspherical manifold with $n=\operatorname{dim} B \geqslant 2$, if the primary obstruction $o_{n}(f, \bar{b})=0$, then $f$ cannot be a manifold fibration. 
For $n \geqslant 3, o_{n}(f, g)=0$ iff $\exists f^{\prime} \sim f, g^{\prime} \sim g$ such that $C\left(f^{\prime}, g^{\prime}\right) \cap E^{(n)}=\emptyset$. For $n=2, o_{2}(f, g)$ is just the abelianized obstruction so that $o_{2}(f, g)=0$ does not guarantee that $f$ and $g$ are deformable to be coincidence free on the $n$-th skeleton. Therefore, as already pointed out in Remark 2.2, the following result is a consequence of and not equivalent to Theorem 2.4. We present a simpler and direct proof of this result, as suggested by the generous anonymous referee.

Corollary 2.5. Given a manifold fibration $f: E \rightarrow B$ where $B$ is aspherical with $n=\operatorname{dim} B \geqslant 2,\left.f\right|_{E^{(n)}}: E^{(n)} \rightarrow B$ is surjective.

Proof. Let $\eta: B^{\prime} \rightarrow B$ be the universal cover and $f^{\prime}: E^{\prime} \rightarrow B^{\prime}$ be the pull-back of $f: E \rightarrow B$ by $\eta$. Since $B$ is aspherical, $B^{\prime}$ is contractible and hence $E^{\prime}$ is equivalent to the trivial fibration $B^{\prime} \times F$. By duality, $H_{c}^{n}\left(B^{\prime}\right) \cong \mathbb{Z}$ and $f^{\prime *}: H_{c}^{n}\left(B^{\prime}\right) \rightarrow$ $H_{c}^{n}\left(E^{\prime}\right)$ is injective, where $H_{c}^{*}$ denotes integral cohomology with compact support. If $K^{\prime}$ denotes the $n$-th skeleton of $E^{\prime}$ then the inclusion $i^{\prime}: K^{\prime} \rightarrow E^{\prime}$ induces an injective homomorphism $H_{c}^{n}\left(E^{\prime}\right) \rightarrow H_{c}^{n}\left(K^{\prime}\right)$. It follows that $f^{\prime} \circ i^{\prime}$ induces a nonzero homomorphism on $H_{c}^{n}$. On the other hand, if $f \circ i: K \rightarrow B$ is not surjective, where $i$ is the inclusion of the $n$-th skeleton $K$ of $B$, then $\eta \circ f^{\prime} \circ i^{\prime}$ is not onto which in turn implies that $f \circ i$ must factor through some subspace $B^{\prime \prime}$ of $B^{\prime}$ with $B^{\prime} \backslash B^{\prime \prime} \cong \operatorname{int}\left(D^{n}\right)$. By duality, it is easy to see that $H_{c}^{n}\left(B^{\prime \prime}\right) \cong 0$. It follows that $\left(f^{\prime} \circ i^{\prime}\right)^{*}: H_{c}^{n}\left(B^{\prime}\right) \rightarrow H_{c}^{n}\left(K^{\prime}\right)$ is zero, a contradiction.

\section{Acknowledgements}

This work was conducted during the second author's visits to São Paulo, October 14-21, 2002, May 12-22, 2003, and April 27-May 4, 2004. The visits were partially supported by a grant from Bates College, the "Projeto temático Topologia Algébrica e Geométrica-FAPESP," the "Projeto 1-Pró-Reitoria de Pesquisa-USP," and the N.S.F.

We would like to thank the referee for useful remarks and for providing the short proof of Corollary 2.5.

\section{References}

[1] B. Eckmann, On complexes with operators. Proc. Natl. Acad. Sci. USA, 39 (1953), pp. 35-42.

[2] E. Fadell and S. Husseini, Fixed point theory for non simply connected manifolds. Topology, 20 (1981), pp. 53-92.

[3] E. Fadell and S. Husseini, The Nielsen numbers on surfaces. Contemp. Math., 21 (1983), pp. 59-98.

[4] F. T. Farrell, The obstruction to fibering a manifold over a circle, Actes du Congrés International des Mathématiciens (Nice 1970) Tome 2, GauthierVillars, Paris, (1971), pp. 69-72.

[5] D. Gonçalves, J. Jezierski and P. Wong, Obstruction theory and coincidences in positive codimension. Acta Math. Sinica, to appear. 
[6] D. Gonçalves and P. Wong, Twisted Serre's spectral sequence and Shapiro's lemma. JP J. Geom. Topol., 5 (2005), pp. 97-101.

[7] E. Spanier, Duality in topological manifolds. Colloque de Topologie Tenu à Bruxelles (Centre Belge de Recherche Mathématiques), (1966), pp. 91-111.

[8] G. Whitehead, Elements of Homotopy Theory, Springer-Verlag, New York, 1978.

Daciberg Gonçalves dlgoncal@ime.usp.br

Departamento de Matemática

IME-USP

Caixa Postal 66.281

CEP 05311-970

São Paulo - SP, Brazil

Peter Wong pwong@bates.edu

Department of Mathematics

Bates College

Lewiston, ME 04240

USA

This article is available at http://intlpress.com/HHA/v8/n1/a9 